\title{
Intraoperative Near-Infrared Fluorescence Tumor Imaging with Vascular Endothelial Growth Factor and Human Epidermal Growth Factor Receptor 2 Targeting Antibodies
}

\author{
Anton G.T. Terwisscha van Scheltinga ${ }^{1,2}$, Gooitzen M. van Dam $^{3}$, Wouter B. Nagengast ${ }^{1}$, Vasilis Ntziachristos ${ }^{4}$, \\ Harry Hollema ${ }^{5}$, Jennifer L. Herek ${ }^{6}$, Carolien P. Schröder ${ }^{1}$, Jos G.W. Kosterink ${ }^{2}$, Marjolijn N. Lub-de Hoog ${ }^{2,7}$, \\ and Elisabeth G.E. de Vries ${ }^{1}$ \\ ${ }^{1}$ Department of Medical Oncology, University Medical Center Groningen, Groningen, The Netherlands; ${ }^{2}$ Department of Hospital and \\ Clinical Pharmacy, University Medical Center Groningen, Groningen, The Netherlands; ${ }^{3}$ Department of Surgery, University Medical \\ Center Groningen, Groningen, The Netherlands; ${ }^{4}$ Institute for Biological and Medical Imaging, Technical University of Munich and \\ Helmholtz Center Munich, Munich, Germany; ${ }^{5}$ Department of Pathology, University Medical Center Groningen, Groningen, The \\ Netherlands; ${ }^{6}$ Optical Sciences Group, MESA + Institute for Nanotechnology, University of Twente, Enschede, The Netherlands; and \\ ${ }^{7}$ Department of Nuclear Medicine and Molecular Imaging, University Medical Center Groningen, Groningen, The Netherlands
}

Fluorescence imaging is currently attracting much interest as a method for intraoperative tumor detection, but most current tracers lack tumor specificity. Therefore, this technique can be further improved by tumor-specific detection. With tumortargeted antibodies bound to a radioactive label, tumor-specific SPECT or PET is feasible in the clinical setting. The aim of the present study was to apply antibody-based tumor detection to intraoperative optical imaging, using preclinical in vivo mouse models. Methods: Anti-vascular endothelial growth factor (VEGF) antibody bevacizumab and anti-human epidermal growth factor receptor (HER) 2 antibody trastuzumab were labeled with the near-infrared (NIR) fluorescence dye IRDye $800 \mathrm{CW}$. Tumor uptake of the fluorescent tracers and their ${ }^{89} \mathrm{Zr}$-labeled radioactive counterparts for PET was determined in human xenograft-bearing athymic mice during $1 \mathrm{wk}$ after tracer injection, followed by ex vivo biodistribution and pathologic examination. Intraoperative imaging of fluorescent VEGF- or HER2-positive tumor lesions was performed in subcutaneous tumors and in intraperitoneal dissemination tumor models. Results: Tumorto-background ratios, with fluorescent imaging, were $1.93 \pm$ 0.40 for bevacizumab and $2.92 \pm 0.29$ for trastuzumab on day 6 after tracer injection. Real-time intraoperative imaging detected tumor lesions at even the submillimeter level in intraperitoneal dissemination tumor models. These results were supported by standard histology, immunohistochemistry, and fluorescence microscopy analyses. Conclusion: NIR fluorescence-labeled antibodies targeting VEGF or HER2 can be used for highly specific and sensitive detection of tumor lesions in vivo. These preclinical findings encourage future clinical studies with NIR fluorescence-labeled tumor-specific antibodies for intraoperative-guided surgery in cancer patients.

\footnotetext{
Received May 18, 2011; revision accepted Jul. 14, 2011.

For correspondence or reprints contact: Elisabeth G.E. de Vries, Department of Medical Oncology, University Medical Center Groningen, Postbus 30.001, 9700 RB Groningen, The Netherlands.

E-mail: E.G.E.de.Vries@umcg.nl

Published online Oct. 11, 2011.

COPYRIGHT @ 2011 by the Society of Nuclear Medicine, Inc.
}

Key Words: molecular imaging; monoclonal antibodies; oncology; intraoperative imaging; near-infrared fluorescence

J Nucl Med 2011; 52:1778-1785

DOI: 10.2967/jnumed.111.092833

$\mathbf{F}$ luorescence imaging is currently attracting much interest as a method for superior intraoperative tumor detection, but most current imaging tracers lack tumor specificity. Therefore, combining know-how obtained from targeted therapies such as the use of monoclonal antibodies and expertise on molecular imaging for targeted tumor tracer development might greatly benefit the patient in such a setting.

Molecular imaging is the in vivo characterization and measurement of biologic processes at the cellular and molecular levels (1). Most experience in translating this approach into the clinic has taken place with PET and SPECT in oncology (2). Initially, the focus in radionuclide imaging was on the visualization of general tumor processes such as glucose consumption with ${ }^{18} \mathrm{~F}-\mathrm{FDG}$ PET and DNA proliferation with $3^{\prime}$-deoxy-3'-18 F-fluorothymidine. However, the availability of novel targeted tracers and new detection systems for clinical use has expanded the potential of molecular imaging even into the intraoperative setting. A wide variety of tumor characteristics can now be visualized using tracers based on monoclonal antibodies (3). We developed the PET radiopharmaceuticals ${ }^{89} \mathrm{Zr}$-labeled bevacizumab to image vascular endothelial growth factor (VEGF) and ${ }^{89} \mathrm{Zr}$-trastuzumab to image human epidermal growth factor receptor 2 (HER2) in tumors and tested them successfully preclinically and clinically (4-7). Bevacizumab is a humanized monoclonal antibody that neutralizes all isoforms of the ligand VEGF-A $(8,9)$; trastuzumab, the antihuman HER2 monoclonal antibody, targets the extracellular domain of HER2 (10). 
Fluorescence imaging in the intraoperative setting has recently entered the clinical testing phase. This technique uses light instead of ionizing radiation, is real-time, provides high-resolution imaging, and is relatively inexpensive $(2,11)$. However, compared with PET, optical imaging does not provide noninvasive whole-body imaging, and there exists a limited penetration depth of the signal. There are currently a few intraoperative imaging systems operational in the clinic $(12,13)$, but there is still an unmet need for tumor-specific detection with tumor-specific fluorescent tracers. Our intraoperative tumor-targeted imaging approach of using a small ligandlike folate linked to a fluorophore (i.e., fluoresceine isothiocyanate), which targets specifically the folate- $\alpha$ receptor in ovarian cancer patients, will provide the proof-of-principle concept (Netherlands Trial Register 1980; http://www.trialregister.nl). However, because of tissue-scattering properties, autofluorescence, and absorption of light in the 400- to 650-nm range, the use of near-infrared (NIR) fluorophores is more attractive for deeper-seated tumors with respect to sensitivity and accuracy. Such a tracer should preferably perform in the NIR fluorescence spectrum, which uses light in the 650- to $900-\mathrm{nm}$ spectrum. In this spectrum, autofluorescence signals are thus minimized, and there is the lowest tissue absorption of light, resulting in optimal tissue penetration (14). However, few NIR fluorescence tracers are currently available for use in the clinic, and all are nontargeted. Recently, various groups have reported experience with the introduction of image-guided surgery for the detection of the sentinel node in breast cancer and cervical cancer patients with the NIR fluorescence tracer indocyanine green $(13,15)$.

The use of tumor-specific targeted intraoperative imaging can potentially be expanded to detect tumor margins, locoregional metastases, and residual disease after surgery. We therefore decided to test whether we could translate the excellent performance of radiolabeled bevacizumab and trastuzumab into optical imaging with a clinically applicable tumor-targeted fluorescent tracer. IRDye $800 \mathrm{CW}$ is a NIR fluorophore with optimal characteristics for clinical use, allowing binding to antibodies when used in its $N$-hydroxysuccinimide (NHS) ester form. A preclinical toxicity study with IRDye $800 \mathrm{CW}$ carboxylate showed no toxicity in doses of up to $20 \mathrm{mg} / \mathrm{kg}$ intravenously or intradermally (16).

To develop IRDye $800 \mathrm{CW}-$ labeled bevacizumab and trastuzumab for specific tumor detection in vivo, we verified the results with ${ }^{89} \mathrm{Zr}$-bevacizumab and ${ }^{89} \mathrm{Zr}$-trastuzumab PET. To this end, in vivo mouse models were used with human tumor xenografts for VEGF imaging and HER2 imaging. Intraoperative surgery with the clinically used NIR camera was performed in both subcutaneous and intraperitoneal dissemination tumor models.

\section{MATERIALS AND METHODS}

\section{Cell Lines}

The human ovarian cancer cell line A2780 (HER2-negative,with high VEGF production) was kindly provided by Dr. Thomas C.
Hamilton (Fox Chase Cancer Center). Development of the luciferase-transfected subline A2780 $0^{\text {luc }+}$ was described by Duiker et al. (17). The SK-BR-3 (HER2-overexpressing) breast cancer and KATO-III (HER2-overexpressing) gastric cancer cell lines were obtained from American Type Culture Collection. The ovarian cancer cell line SKOV-3 ${ }^{\text {luc }+}$ (HER2-overexpressing) was obtained from Caliper Life Sciences. Cell lines were quarantined until screening for microbial contamination and mycoplasm had been performed; these tests were negative. Meanwhile, a reproducible supply of cells was established by cryopreservation.

All experiments were performed within a predefined number of passages. Key features of the cell lines were routinely checked. Growth and morphology of the cells was observed and noted to be consistent with prior descriptions of the lines. A2780 luct cells were cultured in RPMI 1640 (Invitrogen), supplemented with $10 \%$ heat-inactivated fetal calf serum (Bodinco BV) and $2 \mathrm{mM}$ L-glutamine (Invitrogen). SK-BR-3 and SKOV-3 $3^{\text {luc }+}$ were cultured in Dulbecco's modified Eagle medium (Invitrogen) supplemented with $10 \%$ fetal calf serum and KATO-III in RPMI 1640 medium supplemented with $20 \%$ fetal calf serum. All cell lines were cultured at $37^{\circ} \mathrm{C}$ in a fully humidified atmosphere containing $5 \% \mathrm{CO}_{2}$.

\section{Bevacizumab and Trastuzumab Labeling with IRDye $800 \mathrm{CW}$ and In Vitro Evaluation of Fluorescence-Labeled Compounds}

IRDye 800CW-NHS (Li-Cor Biosciences) was coupled to bevacizumab (Roche) and trastuzumab (Roche) according to the manufacturer's protocol. In short, antibodies were reacted with IRDye $800 \mathrm{CW}$ at a molar ratio of 1:4 in phosphate-buffered saline ( $\mathrm{pH} 8.5$ ), which was determined as the optimal ratio. The product was purified by ultracentrifugation with Vivaspin sample concentrators (GE Healthcare). Labeling efficiency and purity were determined by size-exclusion high-performance liquid chromatography, with ultraviolet detector wavelengths at 220, 280, and $790 \mathrm{~nm}$. The labeling efficiency obtained was $85 \%-90 \%$, giving a labeling ratio of an average of 3.5 dyes per antibody, with a purity of greater than $95 \%$ after purification. The stability of bevacizumab- $800 \mathrm{CW}$ and trastuzumab$800 \mathrm{CW}$ was verified by storing the final product in $1 \mathrm{~mL}$ of $0.9 \%$ sodium chloride at $4^{\circ} \mathrm{C}$ and $37^{\circ} \mathrm{C}$ for $7 \mathrm{~d}$. Size-exclusion high-performance liquid chromatography measurements were performed for 1 wk. No measurable decreases in bound IRDye 800CW were found. The binding properties to VEGF of bevacizumab-800CW were evaluated using a VEGF-coated enzyme-linked immunosorbent assay, as described previously by Nagengast et al. (4). Binding of the antibody to the antigen was measured with the Odyssey imaging system (Li-Cor Biosciences). Trastuzumab-800CW binding properties were determined using a competition assay with unlabeled trastuzumab on SK-BR-3 cells stitched to a 96-well plate with poly-L-lysine $(0.01 \% \mathrm{w} / \mathrm{v}$ in water; Sigma-Aldrich). Both bevacizumab-800CW and trastuzumab-800CW showed no decrease in-binding affinity.

\section{Bevacizumab and Trastuzumab Conjugation and ${ }^{89} \mathrm{Zr}$ Labeling}

Conjugation and radiolabeling of bevacizumab and trastuzumab were performed as described earlier $(4,5)$. Briefly, both antibodies were first conjugated with the chelator tetrafluorphenol $\mathrm{N}$-succinyldesferal (TFP- $N$-sucDf), which was kindly provided by Dr. Guus A. van Dongen (VU Medical Center). After conjugation, the product was purified by ultracentrifugation and stored at $-20^{\circ} \mathrm{C}$. In the second step, the conjugated antibodies were 
radiolabeled with clinical-grade ${ }^{89} \mathrm{Zr}$-oxalate (IBA Molecular). Because PET is more quantitative than SPECT, we used ${ }^{89} \mathrm{Zr}$ labeled antibodies instead of the ${ }^{111}$ In-labeled analogs.

\section{IgG Conjugation and ${ }^{111}$ In Labeling}

Human IgG (Sanquin) was used as an aspecific control in the experiments. Human IgG conjugation and labeling were performed as described by Ruegg et al. (18). Briefly, IgG was first conjugated to the bifunctional conjugating agent 2-(4-isothiocyanatobenzyl)-diethylenetriaminepentaacetic acid (p-SCN-Bn-DTPA; Macrocyclics). After conjugation, the product was stored at $-20^{\circ}$ C. Conjugated human IgG was radiolabeled with ${ }^{111} \mathrm{In}$-chloride (Covidien).

Glassware, materials, and solutions used for the ${ }^{89} \mathrm{Zr}$ and ${ }^{111} \mathrm{In}$ conjugation and labeling procedures were sterilized, pyrogen-free, and metal-free. All radiolabeled antibodies had a purity of greater than $95 \%$ before administration to the animals.

\section{Animal Experiments}

For subcutaneous tumor models, cells were harvested by trypsinization and resuspended in phosphate-buffered saline. In vivo imaging and ex vivo experiments were conducted using male nude BALB-c mice (BALB/cOlaHSD-foxn ${ }^{\text {nu}}$ ) obtained from Harlan Nederland. Mice were not kept on a specific diet. At 6-8 wk of age, the mice were injected subcutaneously with $5 \times 10^{6}$ A2780 luc $+(n=10)$ or $2.5 \times 10^{6}$ SK-BR-3 $(n=10)$ cells in $0.3 \mathrm{~mL}$ of Matrigel (BD Bioscience). A2780 luc + was selected for these experiments given its high VEGF tumor levels when xenografted and the characterization of ${ }^{89} \mathrm{Zr}$-bevacizumab available in this model (4). Trastuzumab- $800 \mathrm{CW}$ was tested in the SKBR-3 tumor-bearing mice.

Tumor growth was followed with caliper measurements. The tracer was injected when the tumors measured $6-8 \mathrm{~mm}$ in diameter (for A2780, 2 wk after tumor cell inoculation; for SK-BR-3, 4 wk after inoculation). To compare fluorescence optical imaging with PET, mice received a coinjection of IRDye $800 \mathrm{CW}$ - and ${ }^{89} \mathrm{Zr}$ (5 MBq)-labeled bevacizumab or trastuzumab together with the same protein dose of ${ }^{111} \mathrm{In}-\mathrm{IgG}(1 \mathrm{MBq})$ as an aspecific control. During a scan sequence, images were made immediately after injection of the tracer and at 24, 48, 72, and $144 \mathrm{~h}$ afterward. Two protein doses were tested per tracer: for bevacizumab, 30 and $100 \mu \mathrm{g}$, and for trastuzumab, 50 and $100 \mu \mathrm{g}$. Half of the tracer protein dose was labeled fluorescently, correlating with $0.41,0.68$, and $1.36 \mu \mathrm{g}$ of dye for, respectively, 30, 50, and 100 $\mu \mathrm{g}$ of protein, based on a labeling ratio of 3.5 molecules of dye per antibody. The difference in lowest protein dose used was different for bevacizumab and trastuzumab, because a difference in maximal specific activity could be obtained. Tracer injection was via the penile vein in a volume of $150 \mu \mathrm{L}$.

Animals were imaged using a microPET Focus 220 rodent scanner (CTI Siemens). After image reconstruction, in vivo quantification was performed with AMIDE's a Medical Image Data Examiner software (version 0.9.1; Stanford University) (19). The data were presented as the mean standardized uptake value (SUVmean), which analyzes tumor uptake by comparing it with the total average whole-body uptake. Animals were sacrificed after the last scan, and organs and tissues were excised and weighed. Samples and primed standards were counted for radioactivity in a calibrated well-type LKB-1282 Compu- $\gamma$ system (LKB Wallac) and corrected for physical decay. Ex vivo tissue activity was expressed as percentage of the injected dose per gram of tissue. Harvested tumors were paraffin-embedded for further analysis.
In vivo fluorescence and bioluminescence images were obtained with the IVIS Spectrum (Caliper Life Sciences). Fluorescence images were retrieved by measuring a spectrum with different excitation wavelengths of 675 and $745 \mathrm{~nm}$ and using a filter of $800 \mathrm{~nm}$. Data were analyzed using Living Image 3.2 software (Caliper Life Sciences). To determine tumor-to-background ratio (TBR) from NIR fluorescence images, the tumor boundary was set as a region of interest, and the ratio was calculated by comparing the tumor uptake with the background value within the mouse. The TBR was determined after spectral unmixing of the fluorescence signal, performed by Living Image software, to distinguish the IRDye $800 \mathrm{CW}$ signal from autofluorescence.

Bioluminescence was achieved by injecting the animals intraperitoneally weekly with D-luciferin $(150 \mathrm{mg} / \mathrm{kg}$; Xenogen $)$ reconstituted in phosphate-buffered saline, followed by imaging.

The real-time intraoperative multispectral fluorescence imaging system as developed by Themelis et al. (12) was used to visualize the location of the fluorescence-labeled antibodies. This system contains 3 cameras operating in parallel: a color and fluorescence camera and an intrinsic camera for light attenuation images at the excitation wavelength. Images and videos were obtained during surgery and ex vivo studies. Figures were made with Photoshop (version CS4; Adobe) for creation of the overlay pictures.

Female nude BALB-c mice (BALB/cOlaHSD-foxn ${ }^{\text {nu}}$ ) with A2780 luct $(n=5)$, SKOV-3 luc $+(n=10)$, and KATO-III $(n=5)$ cells were used for intraperitoneal dissemination tumor models. Mice were kept on an alfalfa-free diet to reduce autofluorescence in the peritoneum. A total of $2 \times 10^{6}, 5 \times 10^{6}$, or $2 \times 10^{6}$ cells were injected intraperitoneally (volume, $0.5 \mathrm{~mL}$ ). Tumor growth was tracked with weekly bioluminescence measurements in mice that received cells of a luciferase-positive cell line, using the IVIS Spectrum. The fluorescent tracer was injected intraperitoneally 3 wk after inoculation of A2780 $0^{\text {luc }+}$ and KATO-III and 4 wk after SKOV-3 $3^{\text {luc }+}$. Ten mice inoculated with the A2780 luc + $(n=5)$ or SKOV-3 $3^{\text {luc }+}(n=5)$ received $100 \mu \mathrm{g}$ of bevacizumab$800 \mathrm{CW}$. Trastuzumab-800CW $(100 \mu \mathrm{g})$ was administered in 5 mice inoculated with SKOV-3 $3^{\text {luc }+}$ and 5 mice with KATO-III tumors. On day 4 after tracer injection, all mice received isoflurane inhalation anesthesia (induction, $3 \%$; maintenance, $1.5 \%$ ) to undergo NIR fluorescent imaging during surgery.

To validate the findings with the intraoperative fluorescence imaging system, 1 animal from each group was also scanned in the IVIS Spectrum system. If present, at least 3 fluorescent spots were excised intraoperatively for pathologic and fluorescence microscopy analysis. Harvested fluorescent lesions were paraffinembedded for further analysis. During surgery, intraoperative imaging was performed by recording still images and movies. After this procedure, the mice were sacrificed. The animal experiments were approved by the animal experiments committee of the University of Groningen.

\section{Ex Vivo Tissue Analysis}

Paraffin-embedded subcutaneous tumors and fluorescent intraperitoneal lesions were stained with hematoxylin and eosin (H\&E) and immunohistochemically for VEGF (SC-152; Santa Cruz) or HER2 (CB11; Neomarkers).

Hoechst staining (33258; Invitrogen) was used to visualize nuclei for fluorescence microscopy. Fluorescence microscopy analysis was performed using an Olympus Fluoview 300 confocal scan box mounted on an Olympus IX 71 inverted microscope. The laser source (Coherent; Paladin) produces photons of 532 and 
$1,064 \mathrm{~nm}$. The optical parametric oscillator (APE Berlin) converts photons to longer wavelengths (20). In this setting, the 532-nm photons are converted to $770 \mathrm{~nm}$, serving to visualize the IRDye $800 \mathrm{CW}$ bound to the antibody. Light at $1,064 \mathrm{~nm}$ was used to visualize the Hoechst nuclear staining by 2-photon fluorescence. The filter set consisted of the combination of 2 band-pass filters (850-90m-2p; Chroma) and long-pass emission filters (HQ795LP; Chroma). Image analysis was performed using FV10-ASW (version 1.6; Chroma).

\section{Statistical Analysis}

Data are presented as mean $\pm \mathrm{SD}$. Statistical analysis was performed using the Mann-Whitney test for nonparametric data (Prism, version 5; GraphPad Software). A $P$ value of 0.05 or less was considered significant.

\section{RESULTS}

\section{Dual-Modality VEGF Imaging}

First, we compared the characteristics of fluorescencelabeled bevacizumab with ${ }^{89} \mathrm{Zr}$-bevacizumab (Figs. $1 \mathrm{~A}$ and 1B). In the luciferase-positive tumors, an increasing bioluminescence signal was observed during tumor growth, providing assurance about the vitality of most tumor cells. Quantification of tumor uptake by TBR of the optical fluorescence images revealed an increased signal with a higher fluorescent tracer protein dose of bevacizumab-800CW (Fig. 1A). The TBR was $1.35 \pm 0.11$ in the $30-\mu \mathrm{g}$ bevacizumab group and $1.93 \pm 0.40$ in the $100-\mu \mathrm{g}$ bevacizumab group $(P=0.014)$ at $6 \mathrm{~d}$ after tracer injection. The tumor SUVmean of ${ }^{89} \mathrm{Zr}$-bevacizumab was slightly lower in the $100-\mu \mathrm{g}$ bevacizumab group than in the $30-\mu \mathrm{g}$ group (Fig. 1B). Optical and nuclear imaging showed an increasing tumor signal over time. Specificity of ${ }^{89} \mathrm{Zr}$-bevacizumab tumor uptake was shown as ${ }^{89} \mathrm{Zr}$-bevacizumab tumor uptake ex vivo, compared with ${ }^{111} \mathrm{In}-\mathrm{IgG}$ as nonspecific tumor uptake. There was a $64.7 \%$ increased uptake in the $30-\mu \mathrm{g}$ bevacizumab group $(P<0.0001)$ and 53.2\% $(P<0.0049)$ increased uptake in the $100-\mu \mathrm{g}$ bevacizumab group, compared with the IgG tumor uptake.

\section{Dual-Modality HER2 Imaging}

Next, we compared the characteristics of fluorescencelabeled trastuzumab with ${ }^{89} \mathrm{Zr}$-trastuzumab. Quantification of tumor uptake by TBR of the optical fluorescence images showed a dose-related effect, with $1.67 \pm 0.18$ at $50 \mu \mathrm{g}$ and $2.92 \pm 0.29$ at $100 \mu \mathrm{g}$ of trastuzumab $(P<0.0001)$ (Fig. 2A). The tumor uptake in the low-dose trastuzumab group was already maximal at day 1 after injection. The optimal time point for measuring fluorescence was comparable to VEGF imaging: between 3 and $6 \mathrm{~d}$ after fluorescent tracer injection, based on increasing TBR. The maximum ${ }^{89} \mathrm{Zr}$ trastuzumab tumor uptake, measured with small-animal PET, was similar for the 50- and 100- $\mu$ g trastuzumab groups (Fig. 2B). The specificity of this ${ }^{89} \mathrm{Zr}$-trastuzumab tumor uptake, shown as tumor uptake of ${ }^{111} \mathrm{In}-\mathrm{IgG}$ (coinjected), was less in both groups: $74.0 \%$ in the $100-\mu \mathrm{g}$ protein dose group $(P=0.0016)$ and $91.2 \%$ in the $50-\mu \mathrm{g}$ protein dose group $(P<0.0001)$.

\section{Use of NIR Fluorescence-Labeled Antibodies for Intraoperative Guidance}

Tumor uptake of the fluorescence-labeled antibodies was clearly visible with the intraoperative fluorescent system in

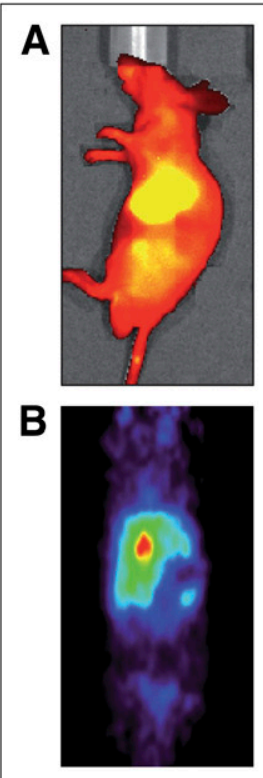

Day 0
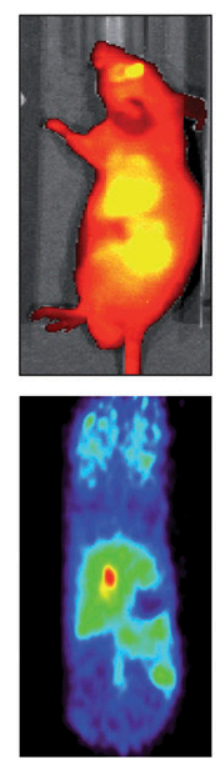

Day 1
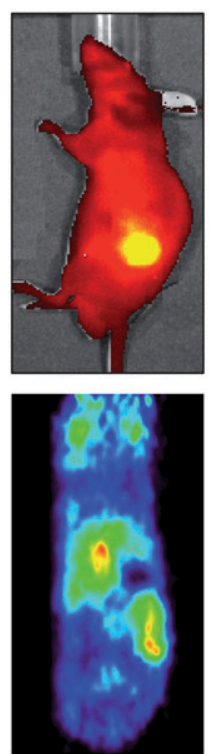

Day 2
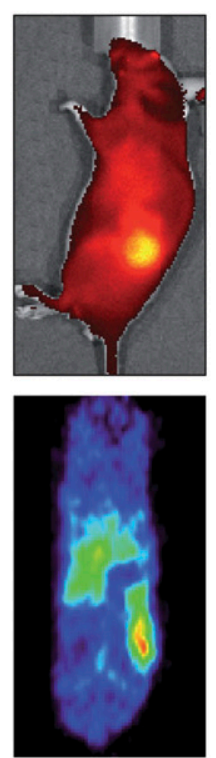

Day 3
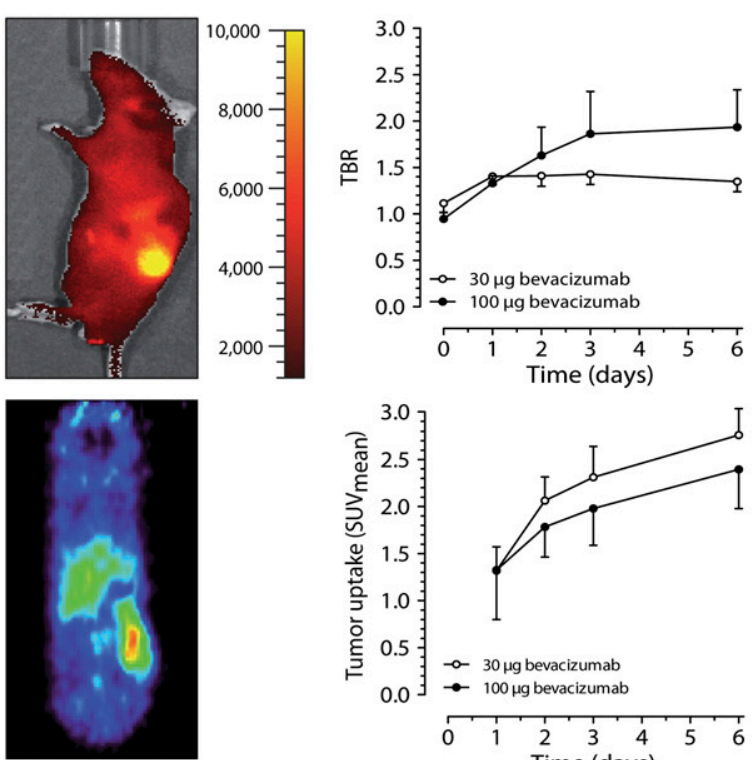

Day 6

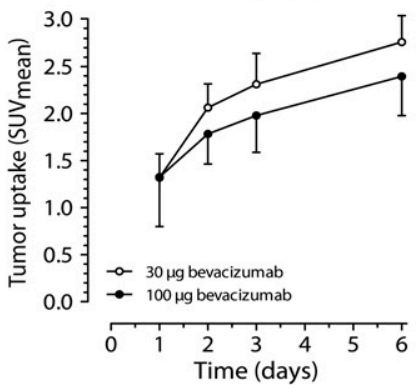

FIGURE 1. Dual-modality imaging of VEGF in subcutaneous A2780luc+ xenograft model. (A) Representative sagittal (2-dimensional) fluorescence images of bevacizumab-800CW immediately after tracer injection of $100 \mu \mathrm{g}$ of bevacizumab and at indicated days, and relative tumor uptake displayed in TBR. (B) Correlated coronal small-animal PET images after ${ }^{89} \mathrm{Zr}$-bevacizumab injection, and tumor uptake (SUVmean) as determined by small-animal PET data quantification for both dose groups (30 and $100 \mu \mathrm{g}$ of bevacizumab). 


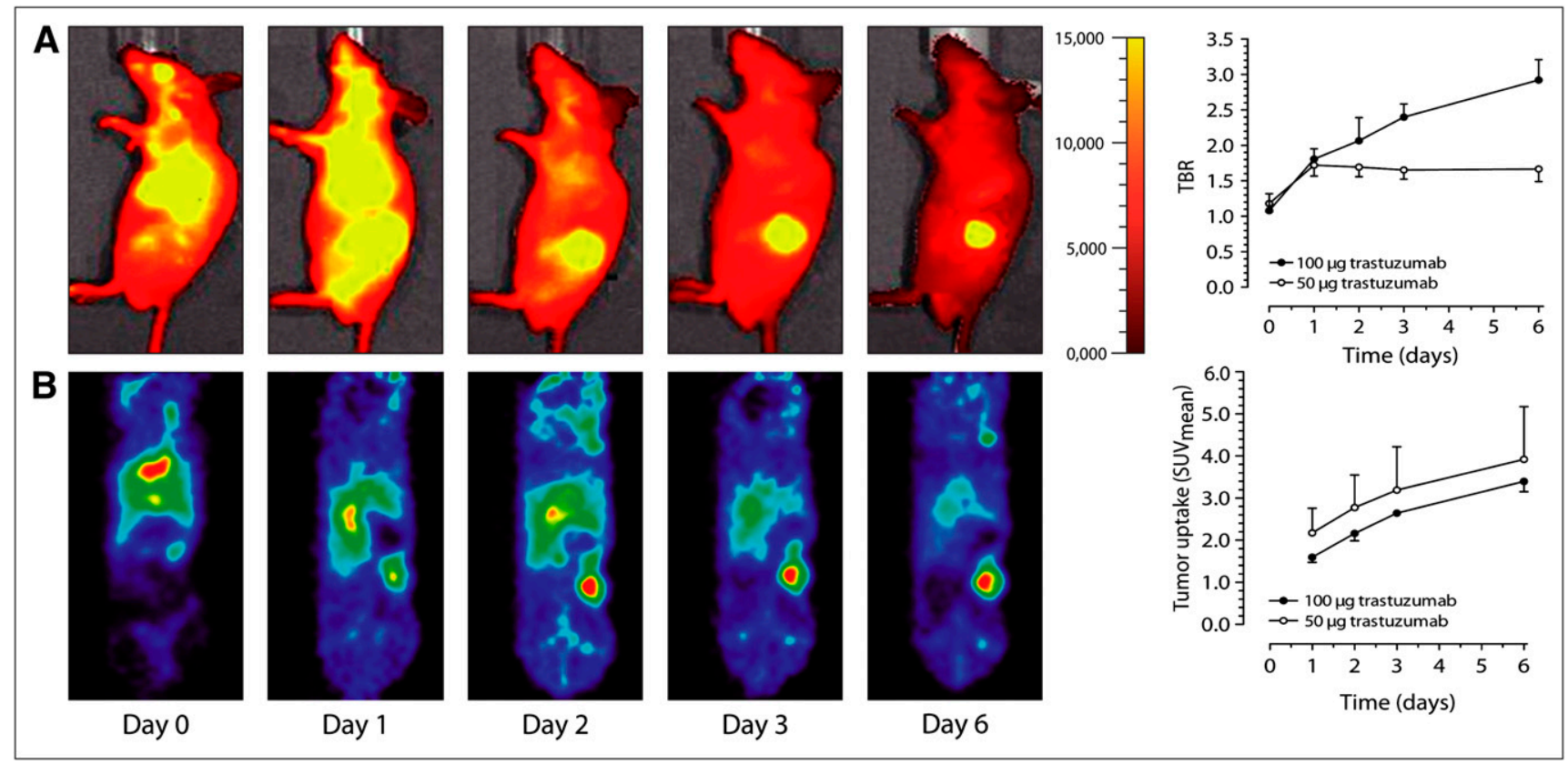

FIGURE 2. Dual-modality imaging of HER2 in subcutaneous SK-BR-3 xenograft model. (A) Representative sagittal (2-dimensional) fluorescence images of trastuzumab- $800 \mathrm{CW}$ immediately after tracer injection of $100 \mu \mathrm{g}$ of trastuzumab and at indicated days, and relative tumor uptake displayed in TBR. (B) Correlated coronal small-animal PET images after ${ }^{89} \mathrm{Zr}$ - trastuzumab injection, and tumor uptake (SUVmean) as determined by small-animal PET data quantification (50 and $100 \mu \mathrm{g}$ of trastuzumab).

mice receiving bevacizumab-800CW (Fig. 3) or trastuzumab-800CW (Supplemental Fig. 1A; supplemental materials are available online only at http://jnm.snmjournals.org). The fluorescence signal of the subcutaneous tumor was detectable despite the intact skin overlying the tumor.

Supplemental Video 1 shows the fluorescence signal of trastuzumab-800CW captured by the intraoperative fluorescence imaging system while the surgeon removes the subcutaneous SK-BR-3 tumor.

Tumor models with high peritoneal metastatic spread were used to investigate whether the fluorescence-labeled antibodies were able to visualize small tumor spots in the peritoneal cavity.

In mice bearing the luciferase-positive cell lines, increasing bioluminescence was observed over time after inoculation, indicating tumor growth and vitality of tumor cells.

For bevacizumab-800CW and trastuzumab-800CW, even submillimeter lesions could be distinguished intraoperatively by the emitted fluorescence signal. Peroperative images for bevacizumab-800CW are shown in Supplemental Figures 2A and 2B. Both ovarian cancer models showed intraperitoneal tumor growth measured with bioluminescence imaging. For the HER2-overexpressing SKOV- $3^{\text {luc }}+$, small tumor lesions could be visualized with bevacizumab800CW (Supplemental Fig. 2B) and with trastuzumab$800 \mathrm{CW}$ (Supplemental Fig. 1B) with the intraoperative camera. Trastuzumab-800CW was also able to visualize the HER2-positive KATO-III tumor lesions (Fig. 4). Lesions were found ranging from submillimeter size to several centimeters. In addition, lesions smaller than $1 \mathrm{~mm}$ were removed from the peritoneum on the basis of the fluorescence signal.

The intense fluorescence signal correlates with tumor lesions as measured with bioluminescence, indicating specific tumor uptake and detection of lesions by fluorescence signal during imaging. The fluorescence signal is present where vital tumor cells are shown using bioluminescent imaging of $\mathrm{A} 2780^{\text {luc }+}$ tumors with bevacizumab-800CW (Supplemental Fig. 3A) and SKOV-3 ${ }^{\text {luc }+}$ tumors with trastuzumab-800CW (Supplemental Fig. 3B).

Supplemental Video 2 shows the experimental setting of intraoperative imaging during surgery on a mouse. The
FIGURE 3. Representative intraoperative images of bevacizumab- $800 \mathrm{CW}$ in subcutaneous A2780 tumor-bearing mouse. Ovarian cancer was targeted with bevacizumab-800CW. Weaker background fluorescence of stomach comes from fluorescence of chlorophyll in food of animals used in these groups. These mice were not kept on alfalfa-free diet.
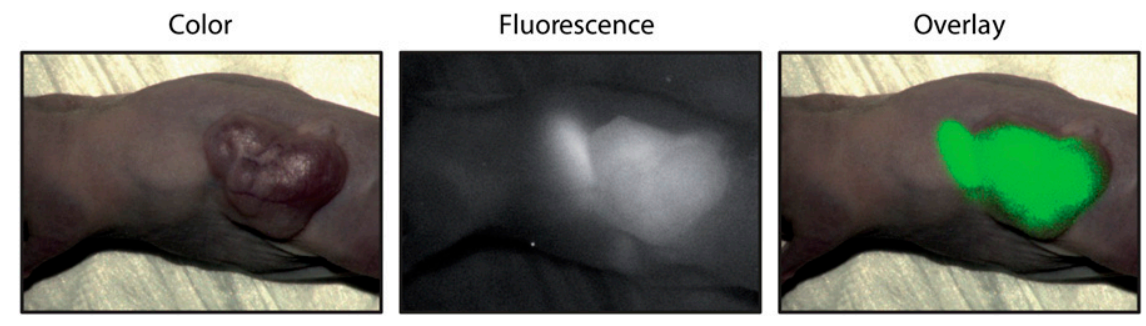

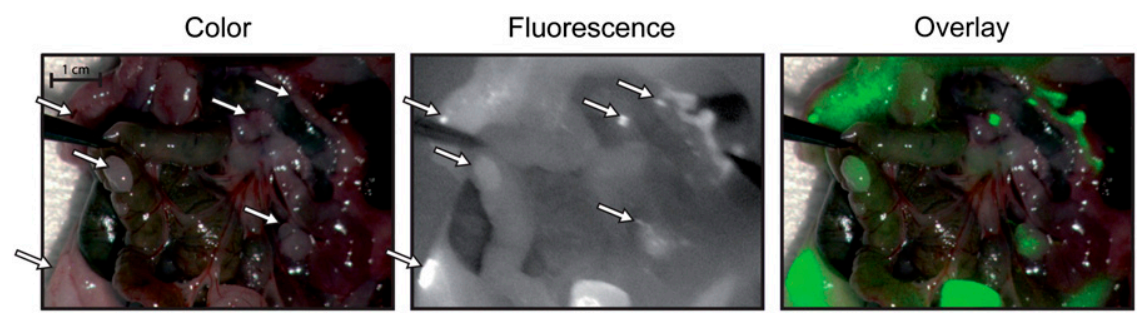

FIGURE 4. Intraoperative images of trastuzumab-800CW in human gastric cancer KATO-III intraperitoneal tumor model.

fluorescence signal of bevacizumab-800CW was captured during removal of intraperitoneal SKOV-3 $3^{\text {luc }+}$ tumor spots as a representative example.

\section{Tumor-Specific Detection with NIR Fluorescence-Labeled Antibodies}

H\&E staining, VEGF staining, and fluorescence microscopy showed that the lesions with fluorescence signals contained tumor cells; this is illustrated for the A2780 luc + subcutaneous tumor in Figure 5. The same results were found in small intraperitoneal lesions of the intraperitoneally injected A2780 $0^{\text {luc }+}$ and SKOV-3 ${ }^{\text {luc }+}$. Comparably, in the SK-BR-3 subcutaneous tumor, viable tumor cells were detected with H\&E, HER2, and fluorescence microscopy (Fig. 5). SKOV-3 ${ }^{\text {luc }+}$ and KATO-III intraperitoneal fluorescent lesions also showed viable tumor cells. As expected, fluorescence microscopy showed bevacizumab$800 \mathrm{CW}$ in the extracellular matrix of the tumor, whereas trastuzumab-800CW fluorescence was detected mainly on the tumor cell surface.

\section{DISCUSSION}

These results are the first, to our knowledge, to show that tumor-targeted NIR fluorescence-labeled therapeutic antibodies can be used for specific tumor detection in vivo in a preclinical setting, using the real-time intraoperative clinical prototype camera system.

In the present study, the excellent selective tumor uptake previously shown with PET of ${ }^{89} \mathrm{Zr}$-bevacizumab and ${ }^{89} \mathrm{Zr}$ trastuzumab in mice bearing human xenografts $(4,5)$ was also observed for the same antibodies labeled with a fluorescent dye.

The TBR obtained for trastuzumab-800CW was somewhat higher than for bevacizumab-800CW. This can at least partially be explained by the fact that bevacizumab targets only human VEGF, which is produced by the implanted human tumor cells. In the human xenograft tumor models, murine VEGF also plays a role, but bevacizumab does not bind or neutralize murine $\operatorname{VEGF}(21,22)$. This assumption is supported by the finding that the radioactive bevacizumab tracer showed a lower tumor uptake in the preclinical setting than in patients $(6,7)$.

${ }^{89} \mathrm{Zr}$-bevacizumab and ${ }^{89} \mathrm{Zr}$-trastuzumab showed high specific tumor uptake, with increasing TBRs during the first $4 \mathrm{~d}$ after tracer injection $(6,7)$. The long half-life of the antibodies implies that these antibodies require days to accumulate optimally in the tumor. Our clinical studies with ${ }^{89} \mathrm{Zr}$-bevacizumab and ${ }^{89} \mathrm{Zr}$-trastuzumab indicated that day 4 after tracer injection is optimal for nuclear imaging. In the present study, the optimal time for optical imaging for both antibodies was around day 3 after fluorescent tracer injection. Our results with the IRDye $800 \mathrm{CW}-$ labeled antibodies directing against VEGF and HER2 revealed the possibility of detecting submillimeter lesions with a clinical intraoperative camera.

This information adds to the recent data obtained for fluorescence-labeled antibodies targeting the epidermal growth factor receptor (EGFR), HER2, and the insulin-like
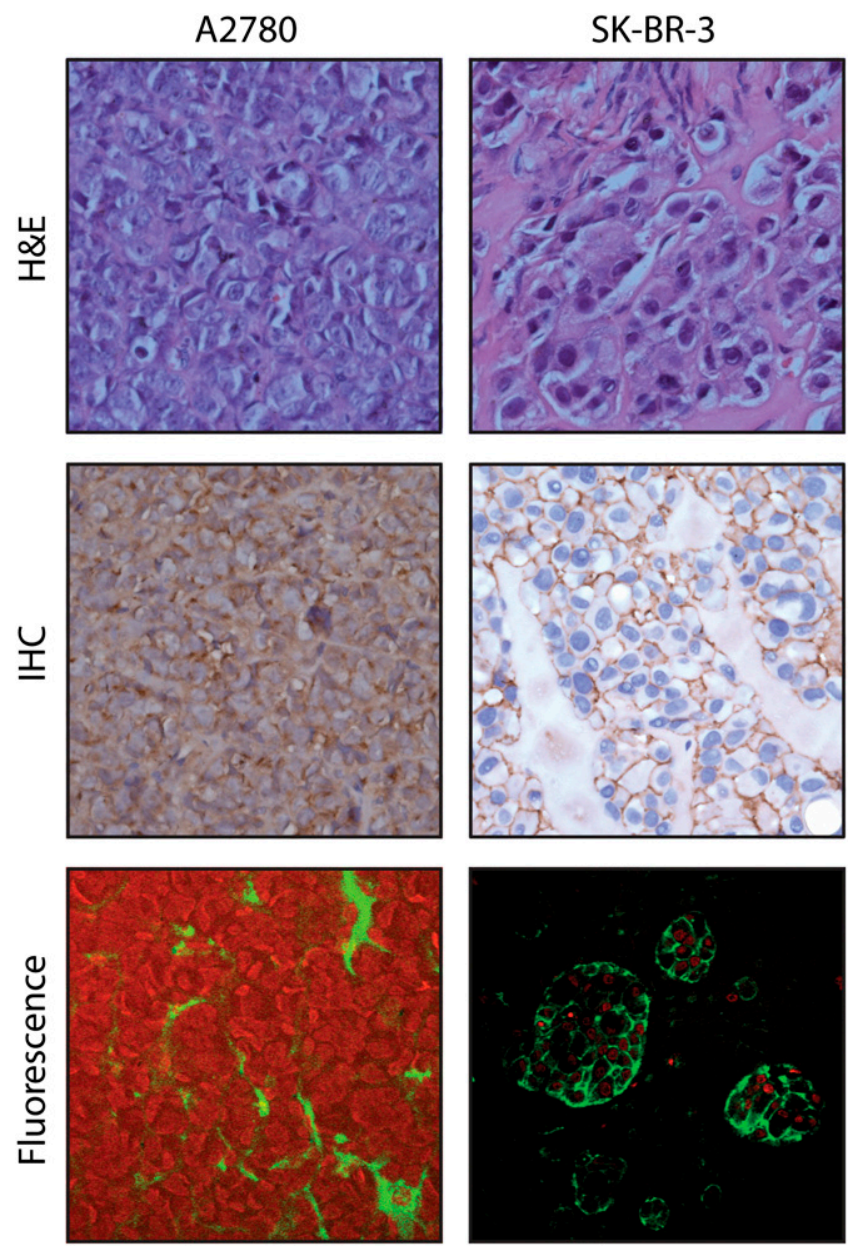

FIGURE 5. A2780 subcutaneous tumor microscopy images $(\times 400)$ showing H\&E staining, VEGF staining, and fluorescence signal of bevacizumab-800CW (green) and nuclei visualized by fluorescence after Hoechst staining (red), and SK-BR-3 subcutaneous tumor microscopy images $(\times 400)$ showing H\&E staining, HER2 staining, and fluorescence signal of trastuzumab-800CW (green) and nuclei (red). IHC = immunohistochemistry. 
growth factor receptor 1 (IGF-1R) (23-26). Cetuximab labeled with the NIR fluorescent dye Cy5.5 showed specific tumor uptake in head and neck squamous cell carcinoma xenografts (23), whereas the IGF-1R targeting antibody AVE-1642 labeled with the NIR fluorescent dye Alexa 680 was specifically taken up by the R-/IGF-1R xenograft tumor (24). Trastuzumab that was dual-labeled with ${ }^{111} \mathrm{In}$ DTPA and IRDye $800 \mathrm{CW}$ showed a similar tumor-tomuscle ratio of 2.25 for fluorescence measurements and 2.66 in a SK-BR-3 ${ }^{\text {luc }+}$ model for SPECT (25). Although these results are promising, no clinical trials have yet been performed with these targeted fluorescent labels. This specific uptake - not only of HER2 and VEGF but also of epidermal growth factor receptor- and IGF-1R-directed antibodies labeled with a fluorescent tracer-further supports the potential to expand our findings to several other targets that could be visualized in tumor tissues of patients with optical imaging when labeled with clinical-grade IRDye 800CW. No NIR-targeted tracers are currently available for clinical use; the tracers discussed in this article could fill this gap in future clinical studies.

Currently, the only NIR fluorescence tracer approved by the Food and Drug Administration for human use is indocyanine green, but it is not a targeted tracer. To address this problem, the antibodies daclizumab (targets CD25), panitumumab (targets HER1), and trastuzumab (targets HER2) were labeled with activatable NIR fluorescent tracers of indocyanine green. They were tested in the preclinical setting and indeed visualized the tumors (26). However, because indocyanine green loses its fluorescence once it is covalently bound to protein, the fluorescence becomes visible only after activation due to cellular internalization of the tracer and degradation of the antibody $(26,27)$. Consequently, this approach cannot visualize VEGF, which is mainly present in the extracellular matrix. This approach could also underestimate the signal, for example, in the case of growth factor receptors such as HER2. In that case, apart from internalization of the receptor, there will also be a receptor at the cell membrane (as demonstrated in fluorescent microscopy results) that will not lead to a fluorescence signal. Our fluorescent microscopic analysis of IRDye $800 \mathrm{CW}$ clearly showed that the signal originates precisely where it was expected for both tracers: for bevacizumab-800CW, between tumor cells in the microenvironment, and for trastuzumab-800CW, intracellularly and on the tumor cell surface.

The dose of labeled antibodies required for molecular imaging in patients is in the subtherapeutic microdose ranges. When antibodies have a linear kinetic profile, no supplementation of unlabeled antibody is necessary for PET. This is the case for U36, a CD44v6-specific murine monoclonal antibody, and for bevacizumab (28-30). Therefore, imaging studies in patients with these ${ }^{89} \mathrm{Zr}$-labeled antibodies were performed with $10 \mathrm{mg}$ for U36 and $5 \mathrm{mg}$ for bevacizumab $(7,31)$. For optical clinical imaging purposes, comparable protein doses will be used. The case is different for trastuzumab, which has dose-dependent pharmacokinetics. After multiple therapeutic doses, trastuzumab clearance has an average terminal half-life of $28.5 \mathrm{~d}$ at steady state (32). However, when given as a single dose of $10 \mathrm{mg}$, trastuzumab has a terminal half-life of only $1.5 \mathrm{~d}$ (33), too short to allow adequate accumulation of the tracer in the tumors and subsequent imaging. In the ${ }^{89} \mathrm{Zr}$-trastuzumab study, supplementation with $50 \mathrm{mg}$ of unlabeled trastuzumab was optimal (6). This aspect will have to be considered for the imaging with trastuzumab-800CW as well.

Given the interesting characteristics of IRDye $800 \mathrm{CW}$ for use in the clinic, we are currently performing the preclinical toxicity testing of bevacizumab-800CW to permit clinical trials with this tracer. However, the best optical imaging may require a cocktail of tracers targeting different tumor characteristics. For example, it is becoming increasingly clear that breast cancer is a heterogeneous disease that can exhibit a variety of characteristics. These experiments indicate that both tracers can be of interest for the clinic, depending on the presence of the target in the tumor. Compared with HER2, VEGF is the more general target for cancer detection, because it is more frequently overexpressed in many tumor types (8).

Apart from intraoperative image-guided surgery, fluorescence imaging systems are also being developed for diffuse optical imaging, hand-held photo-acoustic-based imaging, fluorescent endoscopy, and confocal laser endomicroscopy (34-36). When used for studying breast tissue, diffuse optical imaging is often referred to as optical mammography. This technique is currently being tested in early clinical trials (37). Confocal laser endomicroscopy allows in vivo imaging of the mucosal layer during gastrointestinal endoscopy. Interestingly, the feasibility of VEGF imaging in a preclinical gastrointestinal cancer model and human tissue using confocal laser endomicroscopy was recently demonstrated using a polyclonal rabbit antibody raised against the $-121,-165$, and -89 isoforms of human and murine VEGF labeled with Alexa-Fluor 488 (34). The VEGFspecific signal was visualized and correlated well with ex vivo microscopy. The first clinical trials using confocal laser endomicroscopy with tumor targeted fluorescent tracers are expected to start soon.

\section{CONCLUSION}

In a preclinical setting, NIR fluorescence-labeled antibodies targeting VEGF or HER2 allowed highly specific and sensitive detection of tumor lesions in vivo. These findings strongly support future clinical studies with NIR fluorescence-labeled tumor-specific antibodies in a wide range of clinical applications, including intraoperative image-guided surgery.

\section{DISCLOSURE STATEMENT}

The costs of publication of this article were defrayed in part by the payment of page charges. Therefore, and solely to indicate this fact, this article is hereby marked "advertisement" in accordance with 18 USC section 1734. 


\section{ACKNOWLEDGMENTS}

We thank Martin Jurna, Leila Nahidi Azar, Lucy Crane, Rick Pleijhuis, Esther van Straten, Michiel Bolkestein, Thijs Oude Munnink, and Linda Pot for their technical assistance. This study was supported by grants RUG 20104603 and RUG 2009-4273 from the Dutch Cancer Society. No other potential conflict of interest relevant to this article was reported.

\section{REFERENCES}

1. Weissleder R, Mahmood U. Molecular imaging. Radiology. 2001;219:316-333.

2. Pysz MA, Gambhir SS, Willmann JK. Molecular imaging: current status and emerging strategies. Clin Radiol. 2010;65:500-516.

3. van Dongen GA, Vosjan MJ. Immuno-positron emission tomography: shedding light on clinical antibody therapy. Cancer Biother Radiopharm. 2010;25: 375-385.

4. Nagengast WB, de Vries EG, Hospers GA, et al. In vivo VEGF imaging with radiolabeled bevacizumab in a human ovarian tumor xenograft. J Nucl Med. 2007;48:1313-1319.

5. Dijkers EC, Kosterink JG, Rademaker AP, et al. Development and characterization of clinical-grade ${ }^{89} \mathrm{Zr}$-trastuzumab for HER2/neu immunoPET imaging. $J$ Nucl Med. 2009;50:974-981.

6. Dijkers EC, Oude Munnink TH, Kosterink JG, et al. Biodistribution of ${ }^{89} \mathrm{Zr}$ trastuzumab and PET imaging of HER2-positive lesions in patients with metastatic breast cancer. Clin Pharmacol Ther. 2010;87:586-592.

7. Oosting SF, Nagengast WB, Oude Munnink TH, et al. ${ }^{89} \mathrm{Zr}$-bevacizumab PET imaging in renal cell carcinoma patients: feasibility of tumor VEGF quantification. Eur J Cancer. 2010;7(suppl):72-73.

8. Ferrara N, Davis-Smyth T. The biology of vascular endothelial growth factor. Endocr Rev. 1997;18:4-25.

9. Gerber HP, Ferrara N. Pharmacology and pharmacodynamics of bevacizumab as monotherapy or in combination with cytotoxic therapy in preclinical studies. Cancer Res. 2005;65:671-680.

10. Moasser MM. The oncogene HER2: its signaling and transforming functions and its role in human cancer pathogenesis. Oncogene. 2007;26:6469-6487.

11. Frangioni JV. New technologies for human cancer imaging. J Clin Oncol. 2008;26:4012-4021

12. Themelis G, Yoo JS, Soh KS, Schulz R, Ntziachristos V. Real-time intraoperative fluorescence imaging system using light-absorption correction. J Biomed Opt. 2009; 14:064012

13. Troyan SL, Kianzad V, Gibbs-Strauss SL, et al. The FLARE intraoperative nearinfrared fluorescence imaging system: a first-in-human clinical trial in breast cancer sentinel lymph node mapping. Ann Surg Oncol. 2009;16:2943-2952.

14. Hilderbrand SA, Weissleder R. Near-infrared fluorescence: application to in vivo molecular imaging. Curr Opin Chem Biol. 2010;14:71-79.

15. Crane LM, Themelis G, Pleijhuis RG, et al. Intraoperative multispectral fluorescence imaging for the detection of the sentinel lymph node in cervical cancer: a novel concept. Mol Imaging Biol. 2011;13:1043-1049.

16. Marshall MV, Draney D, Sevick-Muraca EM, Olive DM. Single-dose intravenous toxicity study of IRDye $800 \mathrm{CW}$ in Sprague-Dawley rats. Mol Imaging Biol. 2010;12:583-594.

17. Duiker EW, de Vries EG, Mahalingam D, et al. Enhanced antitumor efficacy of a DR5-specific TRAIL variant over recombinant human TRAIL in a bioluminescent ovarian cancer xenograft model. Clin Cancer Res. 2009;15:2048-2057.
18. Ruegg CL, Anderson-Berg WT, Brechbiel MW, Mirzadeh S, Gansow OA, Strand M. Improved in vivo stability and tumor targeting of bismuth-labeled antibody. Cancer Res. 1990;50:4221-4226.

19. Loening AM, Gambhir SS. AMIDE: a free software tool for multimodality medical image analysis. Mol Imaging. 2003;2:131-137.

20. Jurna M, Korterik JP, Offerhaus HL, et al. Noncritical phase-matched lithium triborate optical parametric oscillator for high resolution coherent anti-Stokes Raman scattering spectroscopy and microscopy. Appl Phys Lett. 2006;89:251116.

21. $\mathrm{Yu} \mathrm{L}, \mathrm{Wu} \mathrm{X}$, Cheng $\mathrm{Z}$, et al. Interaction between bevacizumab and murine VEGF-A: a reassessment. Invest Ophthalmol Vis Sci. 2008;49:522-527.

22. Lee S, Chen TT, Barber CL, et al. Autocrine VEGF signaling is required for vascular homeostasis. Cell. 2007;130:691-703.

23. Rosenthal EL, Kulbersh BD, King T, Chaudhuri TR, Zinn KR. Use of fluorescent labeled anti-epidermal growth factor receptor antibody to image head and neck squamous cell carcinoma xenografts. Mol Cancer Ther. 2007;6:1230-1238.

24. Zhang H, Zeng X, Li Q, Gaillard-Kelly M, Wagner CR, Yee D. Fluorescent tumour imaging of type I IGF receptor in vivo: comparison of antibody-conjugated quantum dots and small-molecule fluorophore. Br J Cancer. 2009;101:71-79.

25. Sampath L, Kwon S, Ke S, et al. Dual-labeled trastuzumab-based imaging agent for the detection of human epidermal growth factor receptor 2 overexpression in breast cancer. J Nucl Med. 2007;48:1501-1510.

26. Ogawa M, Kosaka N, Choyke PL, Kobayashi H. In vivo molecular imaging of cancer with a quenching near-infrared fluorescent probe using conjugates of monoclonal antibodies and indocyanine green. Cancer Res. 2009;69:1268-1272.

27. Kobayashi H, Choyke PL. Target-cancer-cell-specific activatable fluorescence imaging probes: Rational design and in vivo applications. Acc Chem Res. 2011;44:83-90.

28. de Bree R, Roos JC, Quak JJ, den Hollander W, Snow GB, van Dongen GA. Radioimmunoscintigraphy and biodistribution of technetium-99m-labeled monoclonal antibody U36 in patients with head and neck cancer. Clin Cancer Res. 1995;1:591-598.

29. Colnot DR, Quak JJ, Roos JC, et al. Phase I therapy study of 186 Re-labeled chimeric monoclonal antibody U36 in patients with squamous cell carcinoma of the head and neck. J Nucl Med. 2000;41:1999-2010.

30. Gordon MS, Margolin K, Talpaz M, et al. Phase I safety and pharmacokinetic study of recombinant human anti-vascular endothelial growth factor in patients with advanced cancer. J Clin Oncol. 2001;19:843-850.

31. Börjesson PK, Jauw YW, Boellaard R, et al. Performance of immuno-positron emission tomography with zirconium-89-labeled chimeric monoclonal antibody U36 in the detection of lymph node metastases in head and neck cancer patients. Clin Cancer Res. 2006;12:2133-2140.

32. Bruno R, Washington CB, Lu JF, Lieberman G, Banken L, Klein P. Population pharmacokinetics of trastuzumab in patients with HER2 + metastatic breast cancer. Cancer Chemother Pharmacol. 2005;56:361-369.

33. US Food and Drug Administration. FDA Clinical Review of Herceptin 98-0369: Herceptin Trastuzumab (rhuMAb HER2). Available at: http://www.fda.gov/ downloads/Drugs/DevelopmentApprovalProcess/HowDrugsareDevelopedandApproved/ApprovalApplications/TherapeuticBiologicApplications/ ucm091373.pdf. Accessed September 14, 2011.

34. Foersch S, Kiesslich R, Waldner MJ, et al. Molecular imaging of VEGF in gastrointestinal cancer in vivo using confocal laser endomicroscopy. Gut. 2010;59:1046-1055.

35. Ntziachristos V, Razansky D. Molecular imaging by means of multispectral optoacoustic tomography (MSOT). Chem Rev. 2010;110:2783-2794.

36. van de Ven SM, Elias SG, van den Bosch MA, Luijten P, Mali WP. Optical imaging of the breast. Cancer Imaging. 2008;8:206-215.

37. van de Ven S, Wiethoff A, Nielsen T, et al. A novel fluorescent imaging agent for diffuse optical tomography of the breast: first clinical experience in patients. $\mathrm{Mol}$ Imaging Biol. 2010;12:343-348. 